\title{
ИСПОЛЬЗОВАНИЕ МАНУАЛЬНОЙ ТЕРАПИИ В ЛЕЧЕНИИ ПЕРВИЧНОГО НОЧНОГО ЭНУРЕЗА
}

\author{
Сергей Франк, Украина, Киев, МЦ «Украинский Травмоцентр», ортопед-травматолог, \\ мануальный терапевт, Orcid ID: https://orcid.org/0000-0002-0053-6898 \\ Михаил Франк, Украина, Киев, МЦ «Украинский Травмоцентр», Интерн, мануальный \\ mерапевт, Orcid ID: https://orcid.org/0000-0001-5145-9290 \\ Георгий Франк, Украина, Киев, МЦ «Украинский Травмочентр», \\ фельдшер, мануальный терапевт, Национальный медицинский университет \\ uм. О.О. Богомольц̧а, Orcid ID: https://orcid.org/0000-0003-2377-1337.
}

\section{DOI: https://doi.org/10.31435/rsglobal_ws/30112019/6775}

\section{ARTICLE INFO}

Received: 23 September 2019

Accepted: 15 November 2019

Published: 30 November 2019

\section{KEYWORDS}

Primary nocturnal enuresis, manual therapy, functional blockade of spinal motor segments.

\begin{abstract}
Primary nocturnal enuresis is a fairly common disease among children over five years old and while it tends to go away in most of them over time without any treatment, for many, bedwetting continues to be an emotionally stressful and socially destructive state of the early years of their childhood. The presence of a vertebrogenic factor in the clinical picture of primary nocturnal enuresis justifies the use of manual therapy in treating this disease and if the said vertebrogenic factor proves to be the determining cause of the disease, manual correction will allow to quickly, non-invasively, economically, effectively, safely, side-effectlessly and completely eradicate the disease even in cases where treatment by other methods was unsuccessful.
\end{abstract}

Citation: Sergii Frank, Michael Frank, George Frank. (2019) Manual Therapy in Treating Primary Nocturnal Enuresis. World Science. 11(51), Vol.2. doi: 10.31435/rsglobal_ws/30112019/6775

Copyright: (C) 2019 Sergii Frank, Michael Frank, George Frank. This is an open-access article distributed under the terms of the Creative Commons Attribution License (CC BY). The use, distribution or reproduction in other forums is permitted, provided the original author(s) or licensor are credited and that the original publication in this journal is cited, in accordance with accepted academic practice. No use, distribution or reproduction is permitted which does not comply with these terms.

Введение. Ночной энурез (6C00.0 в ICD-11 version 04/2019) - это непроизвольное мочеиспускание во время сна при отсутствии органических заболеваний у детей 5 лет и старше $[1,2,8,20,22]$. Патология достаточно распространена и составляет от 15\% до $20 \%$ среди 5 летних, 5\% среди 10-летних и 1\% среди 15-летних и старше $[1,2,8,20,22,29]$. Мальчики страдают первичным ночным энурезом в 1,5-2 раза чаще, чем девочки $[2,20,22]$. При наличии энуреза в анамнезе у обоих родителей риск его возникновения у ребенка, вне зависимости от его пола, составляет $70 \%$, а при энурезе в анамнезе у одного из родителей - $40 \%$ [2].

Ночное недержание мочи является эмоционально-стрессовым и социально разрушительным состоянием как для страдающих от него детей, так и для их семей $[1,2,8,20,22]$.

Этиология первичного ночного энуреза неясна $[2,20,22]$. Причины энуреза связывают с физиологическими, психологическими, генетическими, неврологическими задержками развития, наследственной предрасположенностью, врожденным нарушением формирования условного «сторожевого» рефлекса, нарушением секреции антидиуретического гормона вазопрессина, а также гормонов регулирующих экскрецию воды и солей, нарушением сна, психологическими проблемами, функциональной емкостью малого мочевого пузыря, потреблением продуктов или напитков с мочегонным эффектом [2, 20, 22, 28]. Ночной энурез также может быть вызван тяжелым храпом или апноэ во сне, в связи с аденотонзиллярной гипертрофией $[4,7,19]$.

Лечение первичного ночного энуреза включает:

- фармакологические вмешательства $[15,16,22]$

- простые и сложные поведенческие вмешательства [5, 17, 18, 22]

- иглоукалывание [20, 22] 
- мануальную терапию и хиропрактику $[3,6,14,20,22,27,30]$

- диетическое и ограниченное питание $[20,22]$

- гомеопатию $[20,22]$

- гипноз [20, 22]

- клофелин (клонидин) [23, 24]

- экстракорпоральную магнитно-иннервационную терапию [23]

- магнитную стимуляцию крестцового корня [23]

- быстрое расширение неба [23]

- тонзиллэктомию [23]

- чрескожную электрическую нервную стимуляцию [23]

Принято считать все методы лечения первичного ночного энуреза, кроме фармакологических и поведенческих вмешательств, экспериментальными и исследовательскими, поскольку их эффективность по этому показанию не установлена [23]. Следует отметить, что ежегодно $15 \%$ детей избавляются от первичного ночного энуреза без какого-либо лечения $[9,22]$, в конечном итоге со временем он проходит самопроизвольно у подавляющего большинства детей [23].

В ряду других экспериментальных и исследовательских методов лечения первичного ночного энуреза используются мануальная терапия и хиропрактика, которые путем манипуляций призваны устранять блокирование (в терминологии мануальной терапии) или подвывихи (в терминологии хиропрактики), возникающие в суставах позвоночника и являющиеся одной из причин появления данной патологии $[3,14,20,22,27,30]$.

Применение мануальной терапии при лечении первичного ночного энуреза обусловлено тем, что в клинической картине заболевания присутствует вертеброгенный фактор и функциональная блокада, т.е. ограничение подвижности сустава функционального характера в пределах нормальной физиологической его функции.

Акт мочеиспускания осуществляется согласованной деятельностью мышц, получающих как соматическую иннервацию (наружный сфинктер мочеиспускательного канала), так и вегетативную. Помимо этих мышц в акте произвольного мочеиспускания принимают участие и мышцы передней брюшной стенки, тазового дна, диафрагмы. Механизм регуляции мочеиспускания включает сегментарный аппарат спинного мозга (Th9-L2, S2-S4), который находится под контролем корковых центров: совместно они реализуют произвольный компонент регуляции [34]. При функциональных нарушениях в сегментарном аппарате спинного мозга может развиться непроизвольное неконтролируемое ночное мочеиспускание.

Хиропрактики в возникновении первичного энуреза усматривают также и влияние диафрагмального рефлекса, образующегося, когда в процессе медленной фазы ночного сна замедляется дыхание и уровень углекислого газа в крови превышает оптимальный. Тогда диафрагмальный нерв, исходящий из сегментов С3-С5, расслабляет гладкую мышечную ткань, в т. ч. мышцу сфинктера, состоящую из гладких мышечных волокон, которая и контролирует открытие и опорожнение мочевого пузыря [31, 32].

Косвенное подтверждение правомерности их предположений можно найти в работах о связи сна с первичным ночным энурезом [7, 19, 21, 32], а также в запатентованном авторском способе лечения энуреза академика PAEH А.Г. Гриценко [Гриценко А.А. (1997) RU2092149C1 - Способ лечения энуреза при функциональных нарушениях сфинктора мочевого пузыря и детрузора].

Цель данного исследования - изучить результаты воздействия мануальной терапии на первичный ночной энурез у детей.

Материалы и методы. Проведен ретроспективный анализ результатов лечения методами мануальной терапии 12 пациентов с первичным ночным энурезом в МЦ «Украинский травмоцентр» в период с января 2018 г. по ноябрь 2019 г. Возраст больных составил от 5 до 11 лет (средний возраст - 7,4 г.). Всем указанным пациентам до обращения в МЦ «Украинский травмоцентр» уже был установлен диагноз первичный ночной энурез в других лечебных заведениях, физические или органические причины ночного энуреза (например, почечная недостаточность, неврологические и психиатрические заболевания, инфекция и т. д.) были исключены. Десять больных проходили безуспешное лечение другими методами, 2 пациента возрастом до 6 лет специализированного лечения не принимали.

Обследование больных в МЦ «Украинский травмоцентр» предполагало проведение осмотра ортопедом-травматологом мануальным терапевтом, а также, при необходимости, для уточнения локализации патологии, выполнение рентгена или МРТ целевых участков 
позвоночника. Мануальная диагностика включала ручную диагностику подвижности и «суставной игры» дугоотростчатых суставов при помощи пассивных движений и смещений в разных плоскостях осей движения позвоночника, специальные приемы исследования функционального состояния мышечной сферы и двигательного стериотипа с целью определения участков функциональной блокады двигательных сегментов позвоночника [33].

Лечение осуществлялось по авторской методике С. Франка [10-13], в основу которой положены лучшие достижения европейской школы мануальной терапии К. Левита [26], развитые и дополненные собственными наработками автора и достижениями украинских коллег. Европейская школа мануальной терапии К. Левита видит предпосылки для мануального лечения в нарушение функции двигательной системы. Наиболее адекватный метод лечения обратимых функциональных ограничений движения - это манипуляции [26, с.20]. Цель манипуляционного лечения состоит в восстановлении функции сустава в тех местах, где она заторможена (блокирована) [26, с.23]. Объектом манипуляций являются функциональные нарушения, в основном блокирования в суставах. Под блокированием понимается ограничение движения в двигательном сегменте [26, с. 20].

Авторская методика С. Франка защищена научными публикациями в европейской научной медицинской периодике [10-13], успешно практикуется на протяжении последних десяти лет и для лечения первичного ночного энуреза у детей в том числе.

Результаты и их обсуждение. Группа исследования была представлена 12 пациентами с первичным ночным энурезом. Возраст пациентов составил от 5 до 11 лет (средний возраст 7,4 г.), группа включала 4 (33,3\%) девочки и 8 (66,7\%) мальчиков. Всем указанным пациентам до обращения в МЦ «Украинский травмоцентр» уже был установлен диагноз первичный ночной энурез в других лечебных заведениях, 10 (83,3\%) из них безуспешно лечились другими методами, 2 (16,7\%) пациента возрастом 5,5 года и 6 лет не проходили специализированного лечения в надежде на скорейшее самопроизвольное устранение данной патологии.

Все 12 больных имели 7 мокрых ночей в неделю с частотой энуреза 1-4 раза за ночь. У 5 больных $(41,7 \%)$ первичный ночной энурез был отягощен запорами, у 4 (33,3\%) хроническими тонзиллитами и аденовирусными инфекциями (один из них перенес аденотонзиллотомию), 2 ребенка (16,7\%) имели апноэ во сне, вызванное аденотонзиллярной гипертрофией. У $3(25,0 \%)$ респондентов наблюдалась положительная семейная история первичного ночного энуреза по линии обеих родителей, у 4 (33,3\%) - по линии одного из родителей. Два пациента (16,7\%) имели лишний вес, один больной (8,3\%) страдал ожирением, четверо $(33,3 \%)$ респондентов имели сколиотическую осанку.

Перед началом лечения у всех пациентов были жалобы на непроизвольное мочеиспускание во время сна с частотой 1-4 раза каждую ночь, которое ребенок не мог контролировать. Поскольку лечение другими методами осталось без ответа, а самопроизвольное избавление от первичного ночного энуреза у этих детей, несмотря на достижение ими возраста приобретения контроля за ночным мочеиспусканием не наступало, то в проводимом исследовании ставилась задача методами мануальной терапии установить контроль ребенка над процессом непроизвольной потери мочи во время ночного сна.

Всем больным проведено первичное лечение, состоящее из 1-10 сеансов мануальной коррекции целевых участков позвоночника. В дальнейшем пациенты наблюдались в МЦ «Украинский травмоцентр» на протяжении 1-2 лет и, по мере необходимости, проходили повторные курсы лечения.

В результате первого курса мануальной терапии состояние $100 \%$ больных улучшилось. Отмечено, что позитивная динамика у пациентов с первичным ночным энурезом зависит как от степени поражения позвоночника, так и от отягощенности анамнеза. Наилучшие результаты получены при лечении первичного ночного энуреза у больных с незначительной механической дисфункцией позвоночника.

Худшие результаты и большая длительность первого и последующих курсов лечения были у детей старшего возраста с отягощенным анамнезом как то: история первичного ночного энуреза у двоих или у одного из родителей, ожирение или избыточный вес пациентов, аденотонзиллярные патологии, апноэ, сколиоз.

После первого курса мануальной терапии у 6 детей (50\%) первичное ночное недержание мочи прекратилось и не возобновлялось больше в течение всего последующего периода наблюдения (2 года), т.е. контроль каждого ребенка над процессом непроизвольной потери мочи во время ночного сна установился на $100 \%$. У двух пациентов $(16,7 \%)$ количество мокрых ночей 
сократилось до одной в неделю, улучшение составило $85,7 \%$. У двоих детей $(16,7 \%)$ количество мокрых ночей сократилось до двух в неделю, улучшение составило 71,4\%. Один пациент $(8,3 \%)$ был мокрым трижды в неделю, наблюдалось улучшение $57,1 \%$. Один ребенок $(8,3 \%)$ продолжал оставаться мокрым 5 дней в неделю, его прогрессивная динамика составила всего лишь $28,6 \%$.

Последующие повторные курсы мануальной терапии после двухмесячного перерыва проводились шестерым пациентам, у которых не удалось достичь полного самоконтроля над процессом непроизвольной потери мочи во время ночного сна. Они состояли из 2-5 сеансов мануальной коррекции проблемных целевых участков позвоночника. В результате еще трое больных, т.е. $25 \%$ от общего количества, полностью избавились от первичного ночного энуреза. У двоих детей, $16,7 \%$ от общего числа, количество мокрых ночей сократилось до 1-2 в неделю, улучшение в результате двух курсов составило 78,6\%. Один ребенок, $8,3 \%$ от общего количества, продолжал оставаться мокрым 3-4 раза в неделю, за два курса его улучшение составило $50 \%$.

Трое пациентов (25\%), которые после двух курсов мануальной терапии продолжали, хоть и не так часто, ночью мочиться в постель, вынуждены были пройти еще через два месяца третий курс мануальной коррекции из 2-5 сеансов. В результате один ребенок, $8,3 \%$ от общего количества, полностью приобрел контроль над процессом непроизвольной потери мочи во время ночного сна, его улучшение составило 100\%. Еще один пациент, $8,3 \%$ от общего количества, продолжал смачивать постель 0-1 раз в неделю - его прогрессивная динамика по результатам трех курсов составила $92,9 \%$. И один мальчик, $8,3 \%$ от общего количества, оставался мокрым 2-3 раза в неделю, по результатам трех курсов его улучшение составило 64,3\%.

Итак, лечение методом мануальной терапии 12 респондентов, страдающих первичным ночным энурезом, позволило за 1-3 курса 10 пациентам (83,3\%) полностью установить контроль над процессом непроизвольной потери мочи во время ночного сна, прогрессивная динамика у одного ребенка составила $92,9 \%$, и еще у одного больного количество сухих ночей достигло $64,3 \%$.

Поскольку лечение первичного ночного энуреза включало мануальную коррекцию всех проблемных участков позвоночника пациентов, то также были отмечены существенные улучшения отягощающих энурез заболеваний, связанных с состоянием позвоночника. Так у троих (75\%) из четверых больных, страдавших запорами, полностью нормализовался стул, у четвертого (25\%) наметилась значительная позитивная динамика. За весь период лечения и последующего наблюдения никто из четверых пациентов (100\%), страдавших ранее хроническими тонзиллитами и аденовирусными заболеваниями, не имел рецидивов данных патологий. У обоих детей (100\%), имевших апноэ во сне, приступы прекратились. Всем четверым пациентам (100\%) со сколиотической осанкой удалось ее исправить.

Полученные результаты дают неоспоримые основания утверждать, что мануальная терапия является действенным методом лечения первичного ночного энуреза в случаях, когда он вызван функциональными отклонениями позвоночника.

\section{Клинический случай №̄1}

Пациентка Б., девочка, 8 лет, школьница, 3 класс. Впервые обратилась в МЦ в январе 2018 г.

$D S$. Первичный ночной энурез.

Жалобы. Непроизвольное не контролируемое мочеиспускание во время ночного сна с частотой 1-2 раза каждую ночь с рождения. Других жалоб нет.

Анамнез заболевания. Самоконтроль мочеиспускания днем во время бодрствования у пациентки установился в возрасте двух лет, навыки пробуждения при позывах к мочеиспусканию во время дневного сна она приобрела к трем годам. Непроизвольная потеря мочи во время ночного сна после достижения ребенком пяти лет продолжалась, потому был установлен диагноз первичный ночной энурез и с семи лет предприняты попытки лечения простыми и сложными поведенческими вмешательствами и психотерапией, которые оказались безрезультатными.

Клинические данныле. Осмотр ортопеда-травматолога мануального терапевта определил участки функциональной блокады двигательных сегментов позвоночника в крестцовой зоне S2-S4.

Лечение. в январе 2018 г. пациентке был проведен один сеанс мануальной терапии, состоящий в мануальной коррекции двигательных сегментов S2-S4 крестцового отдела позвоночника и снятие функционального блока на уровне этих сегментов. Вследствие этого были разблокированы крестцовые парасимпатические нервы, отходящие от данных сегментов и обеспечивающие нервное снабжение детрузора и тригонометрических мышц, контролирующих опорожнение мочевого пузыря. Первичный ночной энурез у ребенка прекратился в ночь после сеанса и не возобновлялся более за весь период наблюдения год и 
десять месяцев. Есть основания утверждать, что энурез пациентки разрешен с помощью манипуляции и результат нельзя отнести к эффекту времени или плацебо.

\section{Клинический случай №2}

Пациент К., мальчик, 10 лет, школьник, 4 класс. Впервые обратился в МЦ в феврале 2018 г.

$D S$. Первичный ночной энурез.

Жалобы. Непроизвольное не контролируемое мочеиспускание во время сна с частотой 1-3 раза каждую ночь с рождения. Периодические головные боли.

Анамнез заболевания. Самоконтроль процесса дневного мочеиспускания и пробуждение при позывах к мочеиспусканию во время дневного сна были приобретены к четырем годам. Непроизвольное бесконтрольное ночное мочеиспускание во время сна с частотой 1-3 раза за ночь после пяти лет продолжалось, потому ребенку был поставлен диагноз первичный ночной энурез. Семья ожидала самопроизвольного разрешения энуреза у мальчика, учитывая положительный опыт данной патологии до 9 лет по линии отца. Поскольку после 9 лет патология не самоустранилась и возникла необходимость социализации ребенка с вынужденным пребыванием его в коллективе в ночное время, то было назначено медикаментозное лечение, которое осталось без ответа, потому в феврале 2018 г. последовало обращение в МЦ «Украинский травмоцентр».

Клинические данные. При осмотре ортопедом-травматологом мануальным терапевтом выявлены функциональные блоки в двигательных сегментах шейного отдела позвоночника С5C7 и пояснично-крестцового отдела позвоночника L3-S1. На рентгенограмме подтверждены данные пальпаторного обследования - функциональные блоки C5-C7 и L3-S1.

Лечение. В январе-феврале 2018 г. проведен первый курс мануальной терапии, включающий 5 сеансов мануальной коррекции функциональных блоков в двигательных сегментах шейного отдела позвоночника C5-C7 и пояснично-крестцового отдела позвоночника L3-S1. Функциональные блоки были устранены и подвижность в указанных сегментах восстановлена. По окончании первого курса мануальной терапии удалось сократить число мокрых ночей с семи до трех, улучшение составило 57,1\%. Периодические головные боли прекратились после первого сеанса мануальной коррекции. В промежутке между первым и вторым курсами соотношение сухих и мокрых ночей в неделю оставалось неизменно стабильным. Второй курс мануальной коррекции был проведен через два месяца и включал два сеанса. В его итоге ребенок оставался сухим все семь ночей в неделю, первичный ночной энурез был излечен на 100\%. По результатам наблюдений свыше полутора лет рецидивов заболевания не последовало.

\section{Клинический случай №3}

Пациент М., мальчик, 11 лет, ученик школы, 5 класс. Впервые обратился в МЦ в январе 2018 г.

$D S$. Первичный ночной энурез. Хронический ринит. Хронический тонзиллит. Сколиоз. Хронический запор. Ожирение.

Жалобы. Непроизвольное не контролируемое мочеиспускание во время сна с частотой 1-4 раза каждую ночь с рождения. Боли в коленях и стопах, быстрая утомляемость. Постоянная заложенность носа, регулярный насморк и боли в горле. С трехлетнего возраста постоянные запоры. Повышенный аппетит.

Анамнез заболевания. Имеет положительную историю первичного ночного энуреза по линии обеих родителей. С рождения и до достижения 11-летнего возраста самоконтроль ночного мочеиспускания не установлен, с пятилетнего возраста предпринимались многократные безуспешные попытки лечения с использованием упражнений для тренировки мочевого пузыря, медикаментозных препаратов, гомеопатии, поведенческой терапии, психотерапии, иглоукалывания. В 9 лет произведена аденотонзиллэктомия, которая лишь незначительно сократила частоту отоларингических заболеваний, но не улучшила течения первичного ночного энуреза. Для борьбы с запорами и избыточным весом с шестилетнего возраста постоянно находится на диетическом питании, занимается лечебной физкультурой, принимает физиотерапевтическое и фармакологическое лечение. Несмотря на предпринимаемые усилия ни один из установленных диагнозов не удалось разрешить, потому в январе 2018 г. пациент обратился МЦ «Украинский травмоцентр».

Клинические данные. Для своего возраста, 11 лет, ребенок отличается крупной комплекцией - рост 165 см, вес - 80 кг, страдает ожирением. При осмотре ортопедом-травматологом мануальным терапевтом определены участки функциональной блокады двигательных сегментов позвоночника: C2-C7, Th2-Th4, Th9-L2, L3-S4. В положении лежа на животе разница в длине ног составила 2 см (правая длиннее левой) за счет блокады в крестцово-подвздошных суставах и сегментах L5-S1, что обусловило контралатеральный левосторонний S-образный грудопоясничный сколиоз, подтвержденный рентгеновским снимком. 
Лечение. в январе-марте 2018 г. пациент прошел первый курс мануальной терапии, состоящий из 8 сеансов мануальной коррекции целевых участков позвоночника. Первым сеансом удалось снять блокаду сегментов С2-С7 шейного отдела позвоночника и обеспечить их частичную подвижность. За два сеанса мануальным воздействием были устранены функциональные блоки крестцово-подвздошных суставов, чем восстановлена одинаковая длина ног в положении лежа на животе. Полностью возвращена подвижность сегментам C2-C7. Также частично возвращена подвижность грудным Th2-Th4 и пояснично-крестцовым L3-S4 сегментам позвоночника. За семь дней между вторым и третьим сеансами больной имел первую в жизни сухую ночь, пробудившись при позывах к мочеиспусканию. Третьим сеансом мануальной коррекции удалось полностью разблокировать сегменты позвоночника C2-C7, Th2-Th4, L3-S4, а также крестцово-подвздошные суставы. Но при этом усилился функциональный блок в сегментах Th10-L2, что вынудило приостановить лечение на два месяца, пока ребенок не подрос на 2 см, за счет чего уменьшился угол перекоса позвонков Th10-L2??? Последующие сеансы с четвертого по восьмой первого курса мануальной коррекции состояли из постепенного устранения функционального блока на уровне сегментов Th10-L2 с увеличением подвижности данных суставов и поддерживающего мануального воздействия на целевые участки позвоночника с целью контроля его функционирования. К концу первого курса число мокрых ночей составило 5 за неделю и сократилось на 28,6\%.

Второй курс лечения был проведен через 2 месяца, состоял из трех сеансов. По его результатам ребенок продолжал оставаться мокрым 3-4 раза в неделю, за два курса его улучшение составило 50\%. Еще через 2 месяца пациент прошел третий курс из двух сеансов, за которые были устранены вновь возникшие функциональные блоки на уровне сегментов Th10-L2. По завершению курса больной оставался мокрым 2-3 раза в неделю, по результатам трех курсов улучшение составило 64,3\%. Общая продолжительность трех курсов лечения составила 1 год и 3 месяца.

Основной целью мануальной коррекции было лечение первичного ночного энуреза, но, вместе с тем, анамнез заболевания был отягощен рядом других патологий, которые удалось устранить с помощью мануальной терапии и, что характерно, более успешно и значительно раньше, чем основное заболевание. Так уже после первого курса ушли отоларингические жалобы - ребенок прекратил страдать хроническими ринитом и тонзиллитом, прошли упорные запоры и ощущение неполного опорожнения кишечника. К концу третьего курса исчезли боли в коленях и стопах, быстрая утомляемость, уменьшился сколиоз. Это объясняется тем, что в процессе лечения мануальному воздействию были подвергнуты не только сегменты позвоночника, связанные с иннервацией мочевого пузыря, а все его проблемные участки, некоторые из них как раз и были коррелированы с указанными патологиями. В то же время, первичный ночной энурез возникает далеко не только от функциональных отклонений позвоночника и, если причина энуреза зависит исключительно от заболеваний позвоночника, то мануальным вмешательством его удается полностью разрешить. Если же в основе этиологии энуреза заложен комплекс причин, связанный не только с позвоночником, то его лечение бывает менее успешным, что и наблюдается в данном конкретном случае.

Выводы. Использование мануальной терапии позволяет оказывать помощь пациентам с первичным ночным энурезом, в том числе и в случаях устойчивых к лечению другими методами. Если в основе этиологии первичного ночного недержания мочи лежат только функциональные нарушения связанных с иннервацией мочевого пузыря сегментов позвоночника, то добиться успеха достаточно просто. Если энурез вызван комплексом причин помимо патологий позвоночника, лечение бывает более продолжительным и менее успешным. В то же время мануальная терапия может быстро, неинвазивно, эффективно, экономично, безопасно, без побочных действий оказывать помощь в борьбе с таким социально разрушительным и эмоционально-стрессовым состоянием, как первичный ночной энурез.

\section{ЛИТЕРАТУРА}

1. Bakker E, van Sprundel M, van der Auwera JC, van Gool JD, Wyndaele JJ: Voiding habits and wetting in a population of 4,332 Belgian schoolchildren aged 10 and 14 years. Scand J Urol Nephrol. 2002, 36 (5): 354362. 10.1080/003655902320783863. Article, CAS, PubMed, Google Scholar

2. Baleva L.S., Kazanskaya I.V., Korovina N.A., Studenikin V.M., Blistinova Z.A., Zavadenko N.N., Zakharova I.N., Novikov P.V., Petrukhin A.S., Proshin V.A., Tamazyan G.V., O.V.Chumakova, Yu.S.Shevchenko, V.K.Tatochenko, Lavrentieva E.B., Doskin V.A., Belousova E.D. (2005) Algorithm of diagnosing and treatment of primary nocturnal enuresis in children. Issues of Modern Pediatrics, Vol. 4, 2: 103-108.

3. Blomerth PR. (1994). Functional nocturnal enuresis. J Manipulative Physiol Ther.; 17(5):335-338. [PubMed] [Google Scholar] 
4. Nesterenko O. V., Goremykin V. I. (2013) Complex approach to treatment of primary monosymptomatic nocturnal enuresis in children. Therapist. Medical scientific and practical portal. 09/13.

5. Caldwell PH, Nankivell G, Sureshkumar P. (2013). Simple behavioural interventions for nocturnal enuresis in children. Cochrane Database Syst Rev.; 7 [PubMed] [Google Scholar]

6. Clar C, Tsertsvadze A, Court R, Hundt GL, Clarke A, Sutcliffe P. (2014). Clinical effectiveness of manual therapy for the management of musculoskeletal and non-musculoskeletal conditions: systematic review and update of UK evidence report. Chiropr Man Therap.; 22:12. [PMC free article] [PubMed] [Google Scholar

7. Sakellaropoulou A. V., Hatzistilianou M. N., Emporiadou M. N. et al. Association between primary nocturnal enuresis and habitual snoring in children with obstructive sleep apnoea-hypopnoea syndrome // Arch. Med. Sci. 2012; 8 (3): 521-527.

8. Fergusson DM, Horwood LJ, Shannon FT: Factors related to the age of attainment of nocturnal bladder control: an 8-year longitudinal study. Pediatrics. 1986, 78 (5): 884-890. CAS, PubMed, Google Scholar

9. Forsythe WI, Redmond A. (1974). Enuresis and spontaneous cure rate. Study of 1129 enuretics. Arch Dis Child.;49(4):259-263. [PMC free article] [PubMed] [Google Scholar]

10. Frank Sergii. (2019) Experience in treating long-term effects of concussions and PTSD using manual therapy combined with shock wave therapy. World Science. 4(44), Vol.2. doi: 10.31435/rsglobal_ws/30042019/6470.

11. Frank Sergii, Frank Michael, Frank George. (2019) Manual Therapy as an Alternative Treatment for Panic Attacks. World Science. 8(48), Vol.2. doi: 10.31435/rsglobal_ws/31082019/6633

12. Frank Sergii, Frank Michael, Frank George. (2019) Treatment of Vertebral Artery Syndrome with Manual Therapy. World Science. 9(49), Vol.2. doi: 10.31435/rsglobal_ws/30092019/6707.

13. Frank Sergii, Frank Michael, Frank George. (2019) Manual Therapy Combined with ShockWave Therapy in Patients with Ankylosing Spondylitis. World Science. 9(49), Vol.2. doi: 10.31435/rsglobal_ws/30092019/6708

14. Gemmell HA, Jacobsen BH. (1989). Chiropractic management of enuresis: time-series descriptive design. J Manipulative Physiol Ther.; 12(5):386-389. [PubMed] [Google Scholar]

15. Glazener CMA, Evans JHC. (2002). Desmopressin for nocturnal enuresis in children. Cochrane Database Syst Rev. ;3 [PubMed] [Google Scholar]

16. Glazener CMA, Evans JHC, Peto R. (2003). Tricyclic and related drugs for nocturnal enuresis in children. Cochrane Database Syst Rev.;3 [PubMed] [Google Scholar]

17. Glazener CMA, Evans JHC, Peto RE. (2004). Complex behavioural and educational interventions for nocturnal enuresis in children. Cochrane Database Syst Rev.;1 [PubMed] [Google Scholar]

18. Glazener CMA, Evans JHC, Peto RE. (2005). Alarm interventions for nocturnal enuresis in children. Cochrane Database Syst Rev.;2 [PubMed] [Google Scholar]

19. Kovacevic L., Jurewicz M., Dabaja A. et al. Enuretic children with obstructive sleep apnea syndrome: Should they see otolaryngology first? // J. Pediatr Urol. 2013; 9 (2): 145-150.

20. Huang T, Shu X, Huang YS, Cheuk DKL. (2011). Complementary and miscellaneous interventions for nocturnal enuresis in children. Cochrane Database Syst Rev. 12 [PubMed] [Google Scholar]

21. Inoue M., et al. Rhythmic slow wave observed on nocturnal sleep encephalogram in children with idiopathic nocturnal enuresis. Sleep. 1987. Vol. 10(6):570-9. Japan

22. Instebo Eigil, Lystad Reidar P. (2016). Chiropractic Care of an 8-Year-Old Girl with Nonorganic, Primary Nocturnal Enuresis: A Case Report. J Chiropr Med. Mar; 15(1): 47-52. Doi: 10.1016 / j.jcm.2016.02.002, PMCID: PMC481201, PMID: 270692

23. Nocturnal Enuresis Treatments (2019). Clinical Policy Bulletins. Number: 0431. http://www.aetna.com/cpb/medical/data/400_499/0431.html

24. Kreitz BG, Aker PD. Nocturnal enuresis: treatment implications for the chiropractor. J Manipulative Physiol Ther. 1994 Sep; 17(7):465-73. https://www.ncbi.nlm.nih.gov/pubmed/7989880

25. Leboeuf C, Brown P, Herman A, Leembruggen K, Walton D, Crisp TC. (1991). Chiropractic care of children with nocturnal enuresis: a prospective outcome study. J Manipulative Physiol Ther.; 14(2):110-115. [PubMed] [Google Scholar]

26. Lewit K., Sachse J., Janda V. (1993) Manual Medicine. - M.: Medicine.

27. Marko RB. (1994). Bed-wetting; two case studies. Chiropr Pediatr.; 1(1):21-22. [Google Scholar]

28. Norgaard JP, Djurhuus JC: The pathophysiology of enuresis in children and young adults. Clin Pediatr. 1993, Spec No 5-9

29. Reed WR, Beavers S, Reddy SK, Kern G. (1994). Chiropractic management of primary nocturnal enuresis. J Manipulative Physiol Ther.; 17 (9):596-600. [PubMed] [Google Scholar]

30. Van Poecke AJ, Cunliffe C. (2009). Chiropractic treatment for primary nocturnal enuresis: a case series of 33 consecutive patients. J Manipulative Physiol Ther.; 32(8):675-681. [PubMed] [Google Scholar]

31. Jouria Jassin M. (2017). Chiropractic Care in Pediatrics. Series on Chiropractic Care. Retrieved from https://nursece4less.com/Tests/Materials/N277BMaterials.pdf

32. Drutz, J., Tu, N. (2017). Patient education: bedwetting in children (beyond the basics). Retrieved from https://www.uptodate.com/contents/bedwetting-in-children-beyond-the-basics

33. Sitel A.B. (2014) Manual therapy (a guide for doctors). - M.: Publishing house BINOM. 468.

34. Ed. by Odinak M.M. (2014) Nervous diseases "SpetsLit" Publishing House, St. Petersburg. 